\title{
Structural Design and Experimentation of Wheeled Home Monitoring Robot with the Obstacle Performance
}

\author{
Wang Zhao, Zhang Yong-de*, Jiang Jin-gang, Chen Zun-yin, Chen Zhu-xiang, Cui \\ Shi-jia, Sun Xue-lian, Zhang Jun and Bi Jin-tao \\ Intelligent Machine Institute, Harbin University of Science and Technology, Harbin \\ 150080 China \\ *zhangyd@hrbust.edu.cn
}

\begin{abstract}
In view of the problems of the home monitoring robot obstacle-navigation performance is poor and obstacle to restore stability is slow, according to the structural characteristics of home monitoring robot, developing a six wheels home monitoring robot, the main features of which lies in the use of independent suspension system combines a double wishbone type and McPherson independent suspension system, effectively improve the obstacle properties and stability of home monitoring robot. Based on the experimental prototype, carried out the experiment of robot obstacle movement, and proved that the structure has the performance of good stability and good obstacle.
\end{abstract}

Keywords: Wheeled home monitoring robot, Structural design, Obstacle performance

\section{Introduction}

Nowadays, due to the increasing pressure of life, people always need for work away on business, so that no one in the state long-term care home, the chance of catch fire, gas leaks and other problems occur increased. In addition, the trend of population aging, empty nesters phenomenon is particularly prominent. Based on the above reasons, people for home monitoring robot needs more and more intense, a variety of home robots emerged. Foreign research in intelligent robot always is a leader, in the field of home service robots, mainly in Japan, the United States and the European research in the majority of developed countries [13]. In 2008, Japan's NEC has released a home-based childcare robot "PaPeRo". Swedish appliance manufacturer company Electrolux developed the first fully automated cleaning robot around the world. In 2009, the famous American robot company WOWWEE new robot "ROVIO" officially landed in China [4], French Robotics firm Aldebaran developed a service robot having a function of emotion recognition "Pepper". In 2011, the United States Schultze WORKS studio designed a production of household robots Luna. However, compared to the domestic robot technology from the world's advanced level there is a gap. In 2005, the service robot market in China is gradually formed the scale, the Advanced Manufacturing Technology Research Institute of CAS Hefei Physical Science Institution development known as "huihui" elderly service robot, Harbin Institute of Technology University developed an intelligent service robot have a remote control and voice recognition function. In April 2012, Ministry of science and technology has issued a service robot "twelfth five-year" special planning and development, and focus on the key technologies of service robotics and industrial development [5-7]. Therefore, the development of a home monitoring surveillance robot has broad market prospects and value. 
In this thesis, analyze the development trend of domestic service robots and market demand, design the mechanical structure of the wheeled home monitoring robot, after analyzing the robot design requirements, studied the structure of each part of the robot by modular approach, and targeted improvements and optimizes the conventional construction, get the design of robot structure, at the same time, through constructing the experimental prototype, test the obstacle-surmounting performance and stability of the robot.

\section{The Design of the Robot}

Analyze the mechanical system's work environment and function are the basis of institution design, based on the analysis of the wheeled home monitoring robot's working environment and features requires, determined the wheeled home monitoring robot have characteristics such as small volume, simple structure, suspension, obstacle good performance, flexible movement, a wide range of monitor and According to the structure to determine the structural parameters of the robot, as shown in Table 1:

Table 1. Robot Mechanism Parameter

\begin{tabular}{|c|c|}
\hline The robot structure index & Peak parameter \\
\hline Robot Overall Dimensions & $500 \mathrm{~mm} \times 500 \mathrm{~mm} \times 300 \mathrm{~mm}$ \\
\hline Vertical detection range & $0.4 \mathrm{~m}$ \\
\hline Load capacity & $1 \mathrm{~kg}$ \\
\hline Gradeability & $30^{\circ}$ \\
\hline Obstacle capability & $2 \mathrm{~cm}, \begin{array}{c}\text { Surmountable indoor cable and } \\
\text { small obstacles }\end{array}$ \\
\hline
\end{tabular}

Wheeled home monitoring robot's working process is by remote control robot complete autonomous cruise and video monitoring in the room. So the robot's mobile mechanism must have an ability to surmount obstacles, at the same time in order to meet a wide range of video surveillance, the camera needs to have two rotational degrees of freedom and a $0.4 \mathrm{~m}$ lift height mobile freedom. Based on these analysis, adopts the modular design concept, the structure of the robot can be divided into the following several modules:

(1) The robot wheeled chassis module, used to implement the robot mobile;

(2) Lifting PTZ camera module, through a combination of rotational freedom and lifting freedom, achieve all-round video surveillance;

(3) Wheel independent suspension system module, used to implementing robot surmount obstacles, also has the vibration reduction function.

\subsection{The Design of the Robot Wheeled Chassis}

Wheeled mobile robot chassis part as the main part of the robot, is of great significance for the design of overall structure, according to the wheel type, number and the different of placement, it offers a variety of wheeled mobile chassis institution. Based on the driving characteristics and stability analysis of single wheel robot, two-wheel robot, three-wheel robot, four-wheel robot and six-wheel robot [8], integrated the needed robot features, adopting six wheel designs. 
Wheels increased can lead to the problems of non-coplanar and poor controllability should be improved on the position and driving way. Moving mechanism wheels arranged in two ways: Matrix and omnidirectional ring. Omnidirectional ring can be achieved anywhere omnidirectional movement, however, its surmounting obstacles capability is poor and control complex. Therefore, adopting the conventional arrangement of a matrix, six wheels left and right sides is symmetrical layout. So as to reduce costs, it is six wheels are selection the criteria off-road wheels.

In order to solve the six wheels no-coplanar caused driving force shortage, adopting six wheels full-drive design. But conventional full-driven design is all independent of each wheel motor drive, the quality and volume of the institution are large, and to control the synchronization accuracy requirement is very high, increase the cost of control part and institution part. Here we adopt synchronous belt drive to ensure synchronization, reducing the number of motors, and reducing the quality, volume and cost of the institution. Using different rotation speed to control the steering, achieve zero rotation radius of turn, this design compared to conventional full drive control is simple. The design is shown in Figure 1.

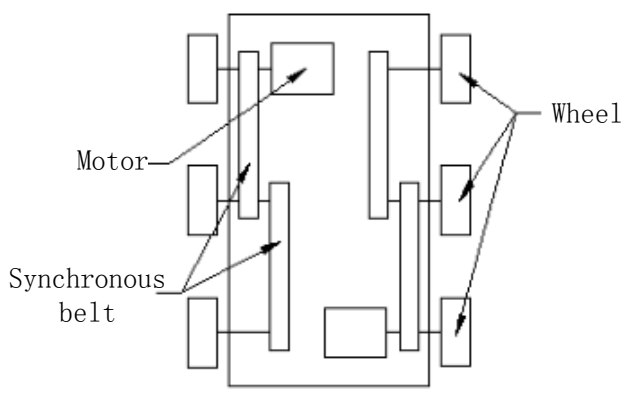

Figure 1. Full Drive Structure

On the basis of the robot design requirements in Table 1, determine the robot chassis size:

(1) Distance between left and right sides of the wheel: $400 \mathrm{~mm}$;

(2) Distance of the same side two wheel center size: $150 \mathrm{~mm}$;

(3) Wheel radius $\times$ wheel width: $50 \mathrm{~mm} \times 60 \mathrm{~mm}$;

(4) Chassis plate size: $400 \mathrm{~mm}$ (length) $\times 200 \mathrm{~mm}$ (width) $\times 3 \mathrm{~mm}$ (thickness).

According to the design size, establish a $3 \mathrm{~d}$ model of the wheeled robot chassis, as shown in Figure 2. Using four sets of timing belt, making the drive force of the two drive motors uniformly transmitted to the six wheels, each wheel is connected with the chassis plate by the same connection, this section connected method details later. There are four support columns on the chassis panel, for supporting the lifting PTZ, front and back two motor to arrangement, save the space, the chassis central space is for battery and control components. 


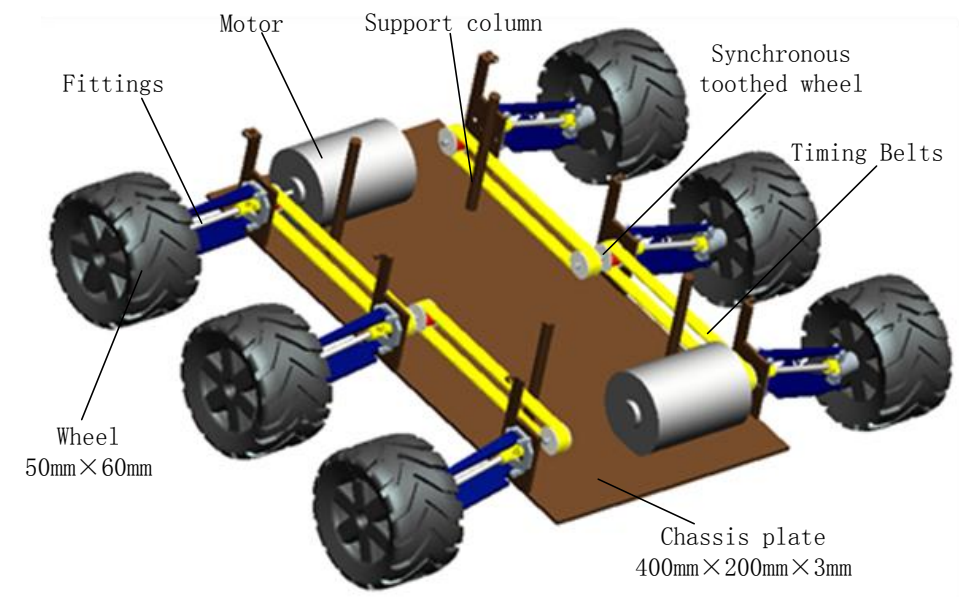

Figure 2. Three-Dimensional Model of Wheeled Chassis

\subsection{The Design of the Lift PTZ}

Robot design requirements have an equipment can realize large range of video monitoring, so it is necessary to analysis the lift PTZ of the robot. Analysis shows that although the robot moving parts can achieve zero radius rotation, however, when the robot steering the body will vibrate, make the stability of the video information reduced. Therefore, to achieve a full range of video surveillance, PTZ mechanism should have a rotational degree of freedom to achieve rotation and a rotational degree of freedom to achieve pitching motion; to expand the scope of monitoring and achieve more parallel perspective position, and it should also have a vertical movement to achieve freedom of movement.

(1) The portion of the rotating head is combined by two servo motor, but considering the servo installation requires a variety of connectors, take up the space is larger, and increased the complexity of the structure. Based on the modular design principle, directly select the products of webcam, as shown in Figure 3. Camera itself has two rotational degrees of freedom, rotation angle of up to $270^{\circ}$, tilt angle greater than $90^{\circ}$, meet the design requirements, and relevant configuration is perfect, it simplifies the complexity of the organization and control.

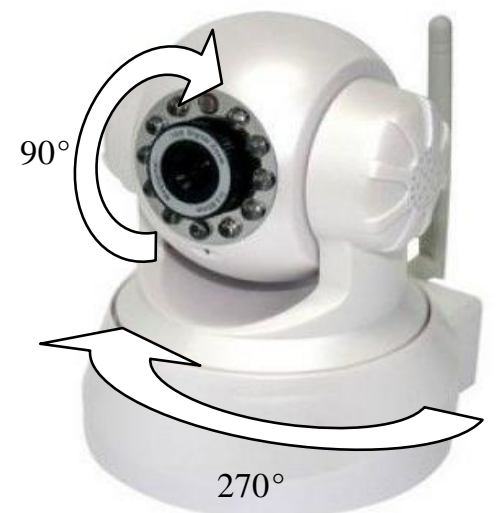

Figure 3. Two Degrees of Freedom Webcam 
(2) The portion of the lifting platform, there are now common lift institution like scissor, sets of cylinder type, column type, crank-type and rail cable type and so on. Which kind of cylinder type and guide wire type belongs to the typical hydraulic lifting platform, does not apply to this research. Shear fork lifting mechanism, by motor driving the screw-way, a plurality of rotating pair combined with exercise, to complete the lifting movement. This mechanism motion stability, take up a small vertical space, but its structure is somewhat complicated, for assembling the demand is higher, and the lifting distance is limited; Pillar type lifting mechanism of electric drive mechanism, consists of sliding nut screw pair, can realize larger distance of lifting movement, and the speed is stability. But the lifting distance is restricted by column of the vertical dimension, cannot achieve full scale movement; Crank arm type lifting mechanism, it is updated form of folding boom type lifting mechanism, have the advantages of occupied a small vertical space and long travel distance, but its stability and control is poor, and require the drive element must have a self-locking feature.

Analysis the design requirements that the lifting mechanism need have a self-locking function, at the same time the structure size and stability are also have certain requirement. Based on the analysis of three feasible solutions, determine the PTZ part adopts shear fork lifting mechanism, adopting the bidirectional self-locking function of the screw, increase the length in order to improve the lifting distance.

Based on the robot design requirements parameter index, detailed analysis the design scheme of the camera lift PTZ, determine the important design dimensions are as follows:

(1) Floor size: $250 \mathrm{~mm} \times 150 \mathrm{~mm} \times 5 \mathrm{~mm}$ (thickness);

(2) Top plate size: $220 \mathrm{~mm} \times 150 \mathrm{~mm} \times 5 \mathrm{~mm}$ (thickness);

(3) Scissors rod size: $150 \mathrm{~mm}$ (bar length) $\times 10 \mathrm{~mm}$ (pole width) $\times 5 \mathrm{~mm}$ (thick bars);

(4) Lead Screw Size: M8 $\times 110 \mathrm{~mm}$ (thread length) $\times 3 \mathrm{~mm}$ (pitch).

According to the design size, using Pro / E 5.0 3D modeling software, building threedimensional model of the robot lift PTZ, shown in Figure 4.

Lifting body set by six groups of $150 \mathrm{~mm} \times 5 \mathrm{~mm}$ scissor lever, each side 3 groups connected by screw, the bottom of scissor lever group and sliding nut connected by revolving joint, drive motor by the belt transmission torque, drive screw is rotated; top plate and the axis of rotation connected by revolute pair, connected to the top plate's guide rail formed a mobile vice.

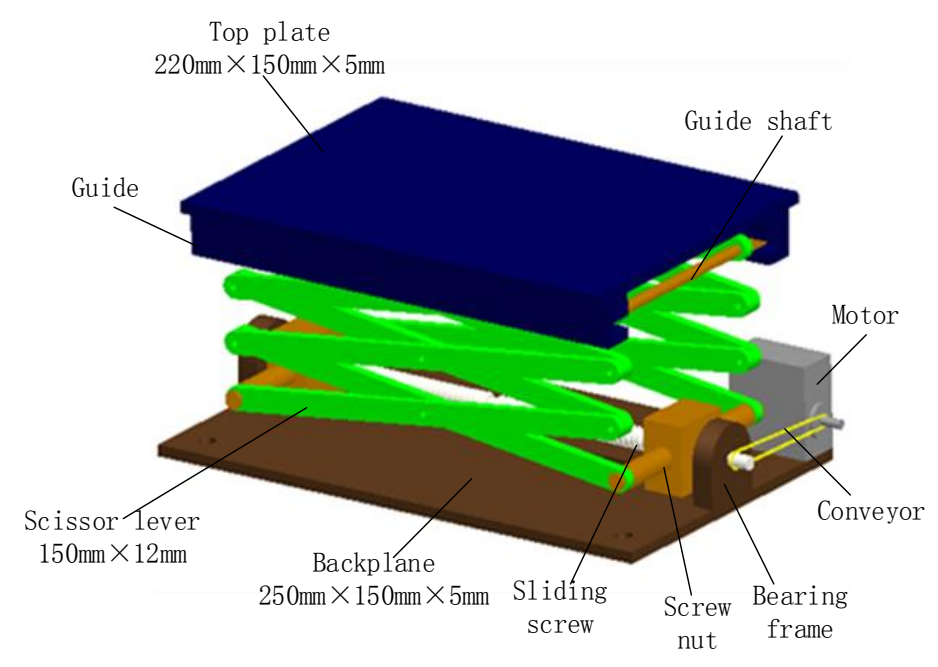

Figure 4. Three-Dimensional Model of the Lift PTZ 
When implementing the lifting movement, the motor-driven double-threaded sliding screw is rotated about its reverse thread, through two nut at the same time, with the same speed relative to one another or move back, change the distance between the two nut to change scissor lever's angle, thereby changing the distance between the floor and the top plate, to achieve the lifting of the goal.

Analysis the motion of scissor lever group, get two extreme positions of the rod set as shown in Figure 5, figure 5 (a) is the minimum lifting height limit position, at this height $\mathrm{H}_{1}$ by lifting the lever itself interference restrictions:

$$
H_{1}=3 \times 10=30 \mathrm{~mm}
$$

Figure 5 (b) is the maximum lifting height limit position, at this height $\mathrm{H}_{2}$ by lifting the lever itself interference restrictions:

$$
H_{2}=3 \times \sqrt{150^{2}-20^{2}}=445.98 \mathrm{~mm}
$$
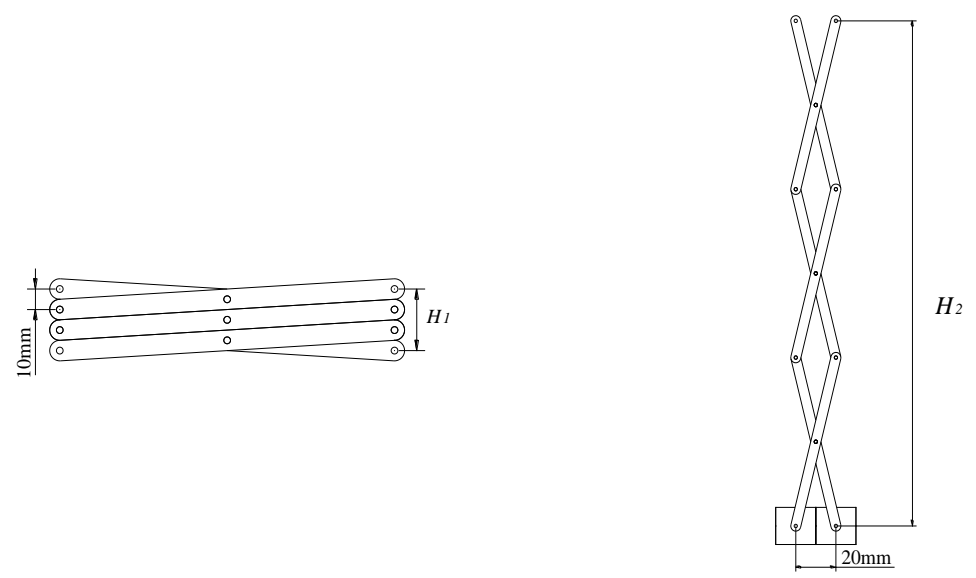
(A) The Minimum Lift Position
(B) The Maximum Lift Position

Figure 5. Scissor Lever Group to Lever Two Limit Position

By the formula (1) and (2) it shows that this lifting mechanism lifting range is:

$$
\Delta H=H_{2}-H_{1}=0.41 m>0.4 m
$$

Therefore, this scheme design meet the robot requirements.

It should be stressed that the lift PTZ must have self-locking function in this design, so here choose sliding screw rather than ball screw, and the screw design parameters meet the requirements of self-locking conditions, namely:

$$
\tan \varphi \leq \mu
$$

Where, $\varphi$ is screw helix angle; $\mu$ is friction angle.

\subsection{Wheel Independent Suspension System Design}

Determined by the above, adopt the whole six-wheel drive scheme as the wheeled mobile robot solution, a multi-wheel structure there is not a co-planarity issues to be resolved. This 
part analysis the vehicle suspension system, solve the wheeled home monitoring robot no coplanarity issues and improve the obstacle-surmounting properties of the design.

Suspension system is consists of spring and shock absorbers, that connected wheel and body, which aims to reduce the car's bumps and improve the vehicle comfortable and stability. The car's suspension system can be divided into non-independent suspension and independent suspension two kinds. Non-independent suspension system both from the same shaft, when one side wheel lifted, the other side of the wheel also will change, making the entire chassis tilt; The independent suspension system, each wheel connected an independent shaft, when one side wheel lifted, the other side of the wheel is not affected, its stability is better than nonindependent suspension systems $[9,10]$.

Since the robot higher requirements for stability, so adopt independent suspension system. Currently, a typical independent suspension system is the following: double-wishbone independent suspension system, McPherson independent suspension system, candle independent suspension system, connecting rod type independent suspension system.

Based on the different characteristics of the suspension system analysis, according to robot design requirements, designed a suspension combined by double-wishbone independent suspension and MacPherson type independent suspension, as shown in Figure 6.

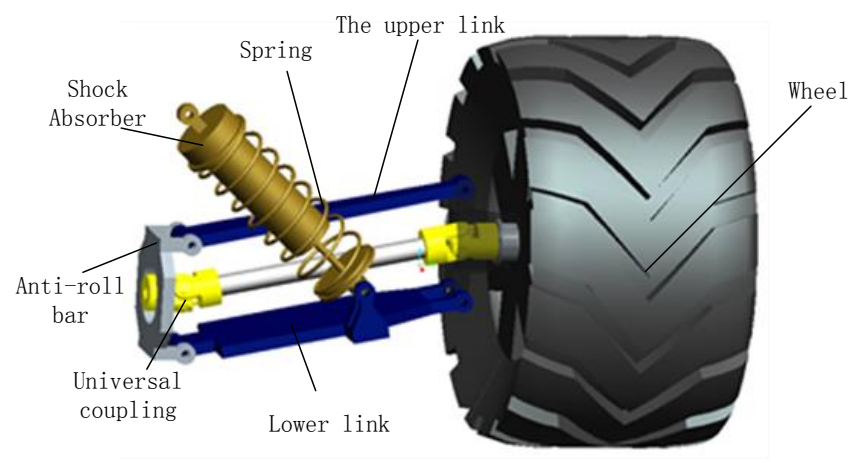

\section{Figure 6. Three-Dimensional Model of Wheel Suspension System}

The suspension system's springs and shock absorbers bottom connected on the lower link, make it not effected by lateral forces, and has the advantage of double wishbone suspension, cornering stability and lateral tilt small; simplified the double-wishbone structure linkage formed into a link plate, has the advantage of McPherson suspension and candle suspension, simple structure, small footprint; used cross-type universal joint, connected the drive output shaft and the wheel axle, so that the driving force can be transmitted through non-linear ways, and this coupler has a small size, transmission ratio is 1 , while meeting the robot simplified structural design requirements.

\subsection{Robot Overall Structure}

Based on the modular design concept, after complete each module's structure analysis and design, and drive element design, combined each module, complete the overall design of the robot, and using Pro / E 5.0 robot modeling, shown in Figure 7.

Using a non-fixed constraint assembly, add drive, through the simulation analysis, the feasibility of the authentication institutions; Analysis of the structure of the interference, the rationality of the authentication institutions; 
Analysis the model quality, verify whether they comply with the design requirements, at the same time measuring the centroid position of the robot, provide parameters for below mechanical analysis. In conclusion, the robot model to meet the design requirements.

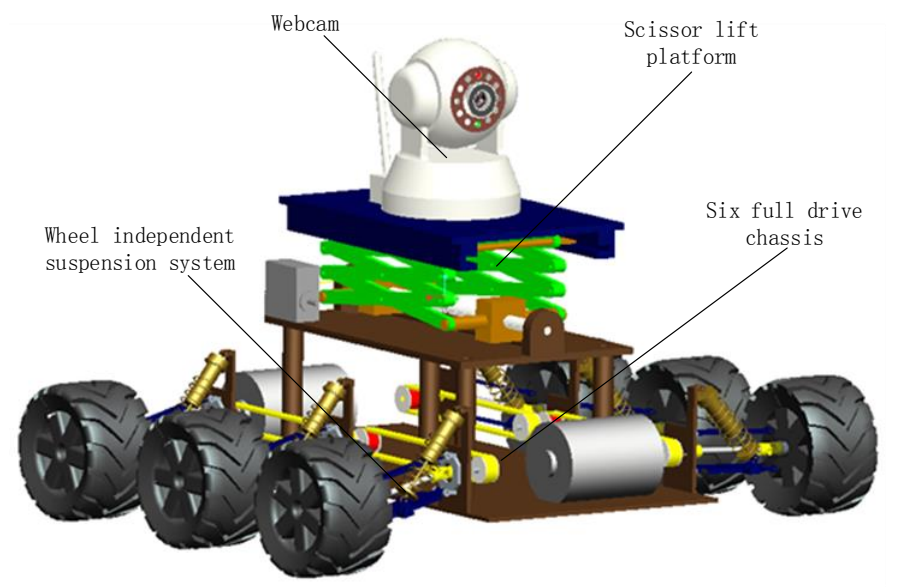

Figure 7. Three-Dimensional Model of the Robot

\section{Prototype Structures and Obstacle Movement Analysis}

Robot prototype consists of PC, based on WIFI control module lower computer and six rounds of home monitoring robot body three parts composed, build experimental prototype as shown in Figure 8.

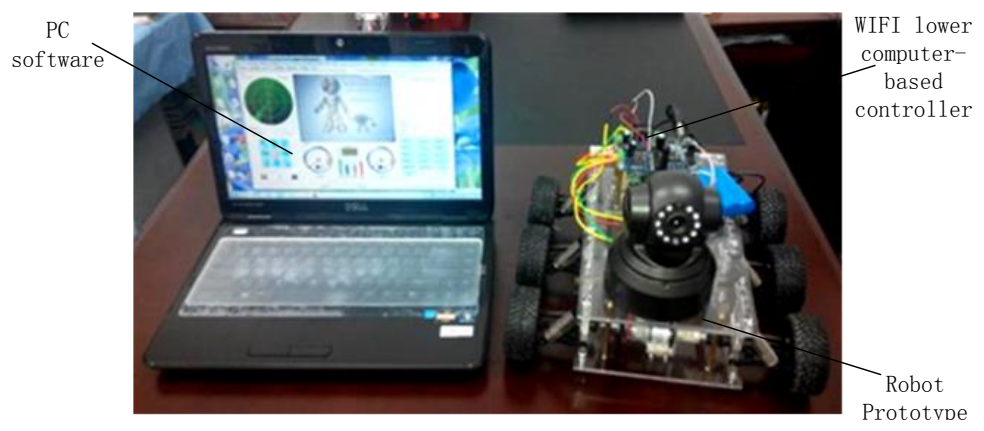

Figure 8. Experimental Prototype Build

Since the robot working environment for indoor, small obstacles encountered, design robot surmounting obstacles campaign, using ground cable cartridge as an obstacle, controls the driving motor driving the robot forward, to verify the achievement of the robot obstacle movement, as shown in Figure 9. 


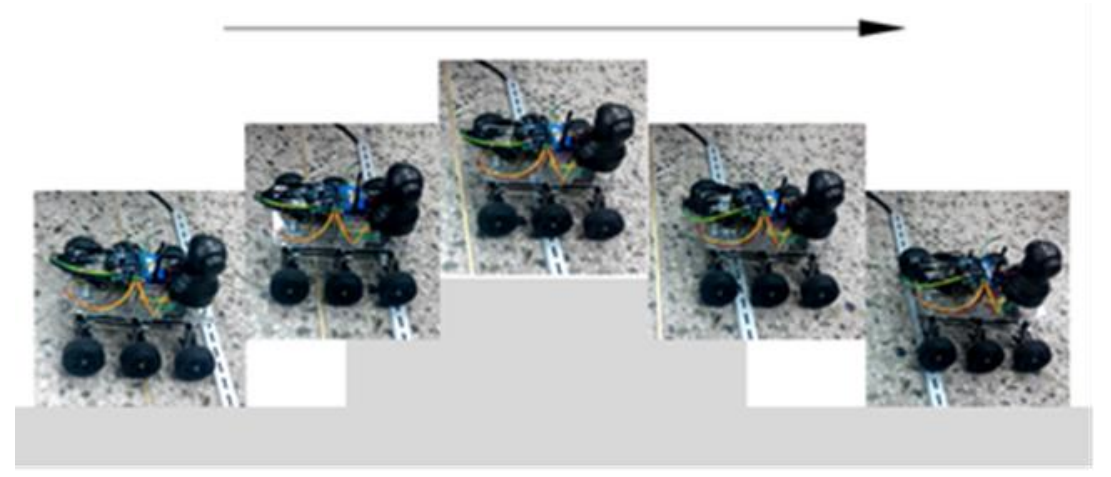

Figure 9. Surmounting Obstacle Exercise Experiment

In process of six home monitoring robot surmounting obstacle, the robot at least to ensure that there are four wheel remains in contact with the ground at the same time, compared with the four-wheel vehicle and other gear train, no tilt in process of surmounting obstacle, smooth running fast recovery after obstacle. The results show that the structure has good stability and good performance obstacle.

\section{Conclusions}

This paper mainly complete wheel home monitoring robot car body design, according to the function of wheeled home monitoring robots that will be divided into a robot wheeled chassis, lift PTZ, the suspension system three different functional modules. By comparison, determined the structure of the scheme to each functional module, and were carried out specific institution design. At the same time, using Pro / E software for all parts of the threedimensional model design, complete 3D assembly, and completed an experimental prototype build, carried out surmounting obstacle movement experiment, determine the structure of the robot meet the requirements of obstacle and stability.

\section{Acknowledgements}

This research was supported by the National College Students Innovation Experiment Program (Grant No. 201310214002).

\section{Reference}

[1] V. Graefe and R. Bischoff, "From Ancient Machines to Intelligent Robots -A Technical Evolution [C]", The Ninth International Conference on Electronic Measurement \& Instruments, 2009 (3):418-431.

[2] T. Fong, I.Nourbakhsh and K.Dautenhahn, "A survey of socially interactive robots [J]", Robotics and Autonomous Systems, 2003,42:143-166.

[3] G. Bekey, "WTEC panel report on international assessment of research and development in robotics [J]",World Technology Evaluation Center, 2006.6.

[4] L.Qiang, "Intelligent household robots [J]", Technology Information, 2013, (22):273-274.

[5] W. Min, Z. Huijie and S. Lin, "Through the patent analysis to see the development of robot technology challenges and opportunities in China [J]", Manufacturing Automation, 2013,(17):5-9.

[6] Z.H. Xin and L. Qingdan, "Robot industry development strategy research in China [J]", China science and technology review, 2012, (04):229-230.

[7] "Service robot "twelfth five-year" development of science and technology special planning [J]", Robot Technique and Application, 2012, (03):1-5.

[8] R. Siegwart and I.R. Nourbakhsh, "Introduction to Autonomous Mobile Robots [M]",MIT ress.2004.

[9] W. Wu, "Suspension System [J]", Shaanxi Auto, 2005, (01):78-83. 
International Journal of Control and Automation

Vol.8, No.11 (2015)

[10] M.D. Nunes, M. Lane and M. Bertoldi, "Dynamic suspension simulation[C]", 12th SAE Brazil Congress and Exposition, Sao Paulo, Brazil, 2003. 